\title{
Acute Psychosis in COVID-19: Is It Due to Favipiravir Treatment or Acute Viral Illness?
}

\author{
Murat Duyan ${ }^{1}$ (D) Ibrahim Ulas Ozturan ${ }^{2}$ (D) \\ Accepted: 4 May 2021 / Published online: 9 May 2021 \\ (C) The Author(s), under exclusive licence to Springer Nature Switzerland AG 2021
}

\begin{abstract}
In this report, we present a case of acute psychosis that occurred after the loading dose of favipiravir, one of the most prescribed antiviral drugs in many countries, in a patient with COVID-19. A 31-year-old female was readmitted to the emergency department after 3 days of sleep disturbance, intermittent paranoid delusions, auditory hallucinations, and agitation that started after the favipiravir treatment was initiated. A physical examination revealed disorganized speech, distorted orientation, and agitation. All laboratory tests, including drug screening and cerebrospinal fluid analysis, were normal. After excluding all the other causes of acute psychosis, favipiravir treatment was discontinued, antipsychotic treatment was started, and the patient was admitted to the psychiatric ward. The symptoms resolved on the second day of hospitalization. Although acute viral illness does seldomly cause psychosis in patients with increased inflammatory response, in the presented case, none of the inflammatory markers were positive, and acute psychosis was attributed to the loading dose of favipiravir. In conclusion, clinicians should be aware of this rare adverse effect and prescribe cautiously to patients at a high risk of psychosis.
\end{abstract}

Keywords COVID-19 · Favipiravir · Psychosis

\section{Introduction}

Favipiravir is a selective RNA polymerase inhibitor that prevents viral replication in human cells [1]. It has been shown that favipiravir can be effective in treating infections caused by RNA viruses such as influenza, Ebola, rabies, norovirus, and SARS-Cov2 [2]. Although the use of favipiravir has been approved in many countries for the treatment of COVID-19 during the pandemic, data regarding the efficacy and safety of favipiravir in COVID-19 is still limited in the literature [3]. In a systematic review and meta-analysis of 9 clinical trials on the efficacy and safety of favipiravir treatment in COVID-19, no significant adverse events were reported in patients under

This article is part of the Topical Collection on COVID-19

Ibrahim Ulas Ozturan

ozturan.iu@gmail.com

Murat Duyan

drmuratduyan@gmail.com

1 Department of Emergency Medicine, Antalya Training and Research Hospital, Antalya, Turkey

2 Department of Emergency Medicine, Mersin Toros State Hospital, Mersin, Turkey favipiravir treatment compared to the control group [3]. In an interim report of an observational study, the most common adverse events described were hyperuricemia (15\%), abnormal liver function (7\%), and diarrhea (1.4\%). While no psychotic symptoms that could have been related to favipiravir treatment have been seen, the agitation has been reported in one patient $(0.05 \%)$ [4]. Another preliminary report of a randomized clinical study showed psychiatric symptoms in 5 patients $(4.3 \%)$ who were under favipiravir treatment [5]. However, it is not clear whether these symptoms were associated with favipiravir treatment or acute viral illness per se. Herein, we present a case of acute psychosis in a patient with mild COVID-19 that progressed gradually over 4 days after receiving a loading dose of favipiravir.

\section{Case Presentation}

A 31-year-old female reporting 5 days of coughing and muscle aches was admitted to the emergency department (ED). The patient had normal vital signs and no remarkable past medical history. Although the patient's physical examination and routine laboratory tests were normal, a computerized tomography of the chest revealed mild peripheral infiltration in 
the right lung. The rRT-PCR examination of a nasopharyngeal swab yielded a positive result for SARS-CoV-2. Diagnosis of COVID-19 was confirmed and favipiravir (1600 mg twice on day 1 and $600 \mathrm{mg}$ twice per day for another 4 days) was prescribed. On the fourth day of treatment, the patient was readmitted to the ED after reporting 3 days of sleep disturbance, intermittent paranoid delusions, auditory hallucinations, and agitation. According to the family, there was no history of trauma, drug or alcohol misuse, or the use of alternative medicine. Upon physical examination, the vital signs were normal, and the Glasgow Coma Scale value was 13 (E4M5V4), but orientation of place, person, and time was distorted. Speech was also disorganized. Further, during the physical examination, the patient became agitated, yelled at the ED staff, and attempted to attack the treating physician. Intramuscular haloperidol (10 $\mathrm{mg})$ and chlorpromazine (25 $\mathrm{mg}$ ) were administrated to sedate the patient. The laboratory tests were within a normal range. A brain CT and an MRI showed no pathology. No progression in pneumonia was detected by the chest X-ray. A urine drug screen was negative. A lumbar puncture was performed to exclude other central nervous system pathologies. Cerebrospinal fluid (CSF) analysis and cultures were negative. The meningitis/encephalitis panel from the CSF was clear. The patient was admitted to the psychiatric department. Favipiravir was discontinued, and risperidone ( $2 \mathrm{mg}$ once per day) and quetiapine (100 $\mathrm{mg}$ once per day) were initiated. The symptoms improved on the second day of admission, and the patient was discharged on the fifth day of hospitalization. The acute onset of psychosis was attributed to favipiravir use. The patient did not have any symptoms after 1-month follow-up.

\section{Discussion}

Although pulmonary and cardiovascular systems are among the most frequently affected systems in SARS-Cov-2 infection [6], prolonged neuropsychiatric or neurodegenerative complications were also reported $[7,8]$. In addition to direct viral cytopathy in the acute phase, cellular and antibodymediated immune response or SARS-CoV-2 neurotropism through the olfactory bulb was hypothesized to be responsible for neuropsychiatric and neurodegenerative disorders in COVID-19 [7, 8]. In another study, it was hypothesized that an interaction between the SARS-CoV-2 proteins and human proteins in the central nervous system mechanisms such as synaptic vesicle trafficking, axonal transport, neurotransmission, or blood-brain barrier elements could be the reasons for neuropsychiatric complications of COVID-19 [9].

Acute psychotic symptoms associated with COVID-19 have been described in several reports $[10,11]$. In these reports, it was postulated that increased inflammatory response resulting from COVID-19 could be the trigger for psychotic symptoms. Interestingly, all previously reported psychosis patients had increased inflammatory markers, especially Creactive protein levels $[10,11]$. In our case, inflammatory markers of the patient were negative upon both ED admissions, yet the psychotic symptoms acutely started on the second day of favipiravir administration. Although it was challenging to determine whether the symptoms were due to the new medication or acute viral illness, the mild course of the clinical findings from COVID-19, having normal laboratory and physical examination findings, suggested that the psychotic symptoms were triggered by the new antiviral regimen.

Many of the drugs used to treat COVID-19 have been shown to be related to neuropsychiatric symptoms, including chloroquine, hydroxychloroquine, umifenovir, interferon-alpha, and corticosteroids [12]. In one study, psychiatric reactions were reported only in five patients $(4.3 \%)$ after favipiravir treatment [5]. However, it is not clear what type of psychiatric symptoms were seen in those patients. To our knowledge, this is the first report that describes a case of acute psychosis in a patient receiving favipiravir treatment.

\section{Conclusion}

Favipiravir may lead to acute psychosis in patients with COVID-19. Clinicians should be aware of this rare side effect and prescribe cautiously, especially to those who have underlying psychiatric disorders.

Author Contribution MD was the treating physicians. MD collected the data. MD and IUO conceptualized the report. IUO drafted manuscript. MD and IUO critically reviewed the manuscript. Both of the authors approved the final version.

Data Availability Not applicable.

Code Availability Not applicable.

\section{Declarations}

Ethics Approval IRB exempt.

Consent to Participate Not applicable.

Consent for Publication Informed consent was obtained from the patient for this case report.

Competing Interests The authors declare no competing interests.

\section{References}

1. Aktaș A, Tüzün B, Aslan R, Sayin K, Ataseven H. New anti-viral drugs for the treatment of COVID-19 instead of favipiravir. J 
Biomol Struct Dyn. 2020:1-11. https://doi.org/10.1080/07391102. 2020.1806112

2. Seneviratne SL, Abeysuriya V, De Mel S, et al. Favipiravir in Covid-19. Int J Progress Sci Technol. 2020;19(2):143-5. https:// doi.org/10.52155/ijpsat.v19.2.1691.3.

3. Hassanipour S, Arab-Zozani M, Amani B et al. The efficacy and safety of favipiravir in treatment of COVID-19: a systematic review and meta-analysis of clinical trials. medRxiv 2021.02.14.21251693. https://doi.org/10.1101/2021.02.14. 21251693.

4. Fujita Health University. Preliminary Report of the Favipiravir Observational Study in Japan. http://www.kansensho.or.jp/ uploads/files/topics/2019ncov/covid19 casereport_en_200529. pdf. Published 2020. Accessed Jan 3, 2021.

5. Chen C, Zhang Y, Huang J, et al. Favipiravir versus Arbidol for COVID-19: a Randomized Clinical Trial. medRxiv 2020.03.17.20037432. https://doi.org/10.1101/2020.03.17. 20037432.

6. Özturan IU, Köse B, Özkan B, et al. Myopericarditis caused by severe acute respiratory syndrome coronavirus 2. Clin Exp Emerg Med. 2020;7(4):326-9. https://doi.org/10.15441/ceem.20.109.

7. Varatharaj A, Thomas N, Ellul MA, Davies NWS, Pollak TA, Tenorio EL, et al. Neurological and neuropsychiatric complications of COVID-19 in 153 patients: a UK-wide surveillance study. Lancet Psychiatry. 2020;7(10):875-82. https://doi.org/10.1016/ S2215-0366(20)30287-X.
8. Duyan M, Ozturan IU, Altas M. Delayed parosmia following SARS-CoV-2 infection: a rare late complication of COVID-19. SN Compr Clin Med. 2021:1-3. https://doi.org/10.1007/s42399021-00876-6.

9. Yapici-Eser H, Koroglu YE, Oztop-Cakmak O, Keskin O, Gursoy A, Gursoy-Ozdemir Y. Neuropsychiatric symptoms of COVID-19 explained by SARS-CoV-2 proteins' mimicry of human protein interactions. Front Hum Neurosci. 2021;15:656313. https://doi. org/10.3389/fnhum.2021.656313.

10. Ferrando SJ, Klepacz L, Lynch S, Tavakkoli M, Dornbush R, Baharani R, et al. COVID-19 psychosis: a potential new neuropsychiatric condition triggered by novel coronavirus infection and the inflammatory response? Psychosomatics. 2020;61(5):551-5. https://doi.org/10.1016/j.psym.2020.05.012.

11. Chacko M, Job A, Caston F, et al. COVID-19-induced psychosis and suicidal behavior: case report. SN Compr Clin Med. 2020;2(11):2391-5.

12. Ghasemiyeh P, Borhani-Haghighi A, Karimzadeh I, et al. Major neurologic adverse drug reactions, potential drug-drug interactions and pharmacokinetic aspects of drugs used in covid-19 patients with stroke: A narrative review. Ther Clin Risk Manag. 2020;16: 595-605.

Publisher's Note Springer Nature remains neutral with regard to jurisdictional claims in published maps and institutional affiliations. 